\title{
Editorial
}

\section{Ionic Liquids: Green Solvents for Chemical Processing}

\author{
Antonia Pérez de los Ríos, ${ }^{1}$ Angel Irabien, ${ }^{2}$ Frank Hollmann, ${ }^{3}$ \\ and Francisco José Hernández Fernández ${ }^{4}$ \\ ${ }^{1}$ Universidad de Murcia, Campus de Espinardo s/n, 30071 Murcia, Spain \\ ${ }^{2}$ Universidad de Cantabria, Avenida de los Castros s/n, 39005 Santander, Spain \\ ${ }^{3}$ Delft University of Technology, Julianalaan 136, 2628 BL Delft, The Netherlands \\ ${ }^{4}$ Universidad Politécnica de Cartagena, C/ Doctor Fleming s/n, 30202 Cartagena, Spain
}

Correspondence should be addressed to Antonia Pérez de los Ríos; aprios@um.es

Received 3 April 2013; Accepted 3 April 2013

Copyright (C) 2013 Antonia Pérez de los Ríos et al. This is an open access article distributed under the Creative Commons Attribution License, which permits unrestricted use, distribution, and reproduction in any medium, provided the original work is properly cited.

Ionic liquids are organic salts, usually consisting of an organic cation and a polyatomic inorganic anion, which are liquid under $100^{\circ} \mathrm{C}$. The most relevant properties of ionic liquids are their almost negligible vapour pressure. Furthermore, their physical and chemical properties can be fine-tuned by the adequate selection of the cation and anion constituents. Ionic liquids have been recognized as environmental benign alternative to volatile organic solvents. Application of ionic liquids in chemical processes has blossomed within the last decade. Indeed, these media have been used in replacement of volatile organic solvents in a wide variety of chemical processes, such as separation and purification, and reaction media in biochemical, and chemical catalysis. This special issue focuses on these topics providing a state-of-the-art overview of new paradigms and challenges in research on ionic liquids as green solvents for chemical processes and highlights the importance of this topic.

In the call for papers, we invited contributions covering all topics in the area, including progress on developing new ionic liquids for use in chemical synthesis and catalysis and recent advances in separation processes using ionic liquids. All contributions were peer-reviewed according to the usual high standards of this journal. Our thanks go to highly qualified and thorough referees that helped us accept seven papers. They greatly contributed to the high quality of the final manuscripts.

The contributions could be grouped into two main research fields where four papers are related to the use of ionic liquid as reaction media and/or catalyst in chemical processes three papers are oriented towards the use of these new solvents on separation processes, specifically as extraction agent in liquid-liquid separations and as liquid phase in supported liquid membranes. In the following, a brief overview and summary of the individual contributions are given.

The first contribution in this issue from V. Srivastava (NIIT University, India) is entitled "ionic-liquid-mediated MacMillan's catalyst for Diels-Alder reaction." A modified and improved protocol for MacMillan's imidazolidinone catalyst for Diels-Alder reaction has been developed using ionic liquid as reaction media. This new protocol allowed to successfully obtain enantiomerically enriched Diels-Alder adducts with high yields and selectivities. The main features of this reaction are as follows (i) the operational simplicity of the procedure, (ii) the cycloaddition adducts were obtained in good yield and selectivities for various dienes with dienophiles resulting in good yields and high selectivity, (iii) obtaining the cycloaddition adduct with low catalyst loading, (iv) the catalyst can be recycled up to six cycles with comparable yields and selectivities, and (v) the protocol was found active in the synthesis of a tedious steroid molecule.

The paper by S. Sajjadifar et al. (Payame Noor University, Iran), which deals with the use of ionic liquid as catalyst in chemical processes, is entitled "1-Methyl-3-(2(sulfooxy)ethyl)-1H-imidazol-3-ium chloride as a new and gGreen ionic liquid cCatalyst for one-pot synthesis of dihydropyrimidinones under solvent-free condition." These authors 
have investigated the use of a Bronsted acidic ionic liquid, 1-methyl-3-(2-(sulfooxy)ethyl)-1H-imidazol-3-ium chloride, as an inexpensive, easy-to-handle, noncorrosive, and environmentally benign catalyst for the Biginelli reaction from an aldehyde, a $\beta$-dicarbonyl, and urea or thiourea. The advantages of the developed procedure are simplicity of operation, very short reaction times compared with other procedures for the preparation of dihydropyrimidinones derivatives, and the high yields of products. Furthermore, the catalyst can be easily recyclable after removing starting materials and water.

The paper "The zwitterionic imidazolium salt: first used for synthesis of 4-arylidene-2-phenyl-5(4H)-oxazolones under solvent-free conditions" by B. Zhou and W. Chen (Zhejiang Sci-Tech University, China) is another example of the successful use of ionic liquid as catalyst. A new imidazolebased zwitterionic-type molten salt was developed and used as catalyst for the synthesis of 4-arylidene-2-phenyl-5(4H)oxazolones, which are very important intermediates for the synthesis of a variety of bioactive molecules and fine chemicals, through the Erlenmeyer reaction under solventfree conditions. The nonhazardous experimental conditions, ease of reaction, short reaction times, high yields, and metalfree catalyst are the notable advantages of this procedure.

In the paper entitled "Cycloaddition reaction of carbon dioxide to epoxides catalyzed by polymer-supported quaternary phosphonium salts" the authors Y. Xiong et al. (Northwest Normal University, China) developed new polymersupported quaternary phosphonium salts (PS-QPS) to be evaluated as catalyst for cyclic carbonates synthesis via the cycloaddition of epoxides with $\mathrm{CO}_{2}$. The results testified that high yields and excellent selectivity can be achieved using PSQPS on the assayed conditions $\left(5 \mathrm{MPa} \mathrm{CO}, 150^{\circ} \mathrm{C}\right.$ in $6 \mathrm{~h}$ ). In addition, the catalyst can be easily separated from the products and reused for up to five times without considerable decrease in the yield of cyclic carbonate.

The first contribution presented in this special issue in the field of the use of ionic liquid in separation processes is from Y. F. Li et al. (Peking University, China) with the title "Partitioning of cephalexin in ionic liquid aqueous two-phase system composed of 1-butyl-3-methylimidazolium tetrafluoroborate and $\mathrm{ZnSO}_{4}$." In this paper, an ionic liquid aqueous twophase system (ILATPS) was applied in the extraction and separation of an hydrosoluble antibiotic, cephalexin (CEX). They found that the distribution of CEX in the ILATPS was influenced by the volume of $[\mathrm{bmim}]\left[\mathrm{BF}_{4}\right]$, the concentration of $\mathrm{ZnSO}_{4}$, temperature, $\mathrm{pH}$, and the volume of $\mathrm{ZnSO}_{4}$ solution. High extraction efficiencies (>92\%) were reached using this approach.

X. Sun et al. (Mudanjiang Normal University, China) contribute to this issue of the journal with their paper "Ultrasonic-assisted extraction of procyanidins using ionic liquid solution from Larix gmelinii bark." They propose a novel extracting method for procyanidins from L. gmelinii bark based on the use of ionic liquids in ultrasound-assisted extraction. The results indicated that the characteristics of anions had remarkable effects on the extraction efficiency of procyanidins. The optimum conditions for the extraction were as follows: [bmim][Br] concentration $1.25 \mathrm{M}$, soak time
$3 \mathrm{~h}$, solid-liquid ratio $1: 10$, ultrasonic power $150 \mathrm{~W}$, and ultrasonic time $30 \mathrm{~min}$. Relative to other methods, the proposed approach provided higher extraction efficiency and obviously reduced energy-consumption time. The method may also prove useful in the development of energy saving and environmentally friendly extraction methods for procyanidins from other plant materials.

The last contribution in this special issue is from I. Cichowska-Kopczyńska et al. (Gdansk University of Technology, Poland) and entitled "Influence of ionic liquid structure on supported ionic liquid membranes effectiveness in carbon dioxide/methane separation." This paper evaluates the potential application of imidazolium ionic liquids containing alkyl fluoride anions (bis (trifluoromethylsulfonyl) imide and trifluoromethanesulfonate) immobilized in polymeric supports (supported ionic liquid membranes) in $\mathrm{CO}_{2}$ separation from gaseous streams. Authors found that the assayed supported ionic liquid membranes were stable, especially when using polypropylene support. The pure gas permeation results showed that the increase in alkyl chain length and therefore increase in viscosity of ionic liquid cause the decrease in permeation values. Furthermore, lower permeability was observed for trifluoromethanesulfonate anion, mainly due to higher viscosity and lower solubility of carbon dioxide. It is worthy to highlight that the SILMs used in this study were highly $\mathrm{CO}_{2}$ selective in $\mathrm{CO}_{2} / \mathrm{CH}_{4}$ system which indicated the possibility of SILMs application in selective separation of carbon dioxide, especially from gas mixtures containing methane such as biogas streams.

The collection of works in this special issue constitutes one more step forward in the race for the development of desired, greener, more sustainable chemical processes in which the ionic liquids have demonstrated to be ver promising green solvents. We hope that you find these papers interesting and wish you much success in your research in the field of ionic liquids as green solvents for chemical processing.

Antonia Pérez de los Ríos Angel Irabien Frank Hollmann Francisco José Hernández Fernández 

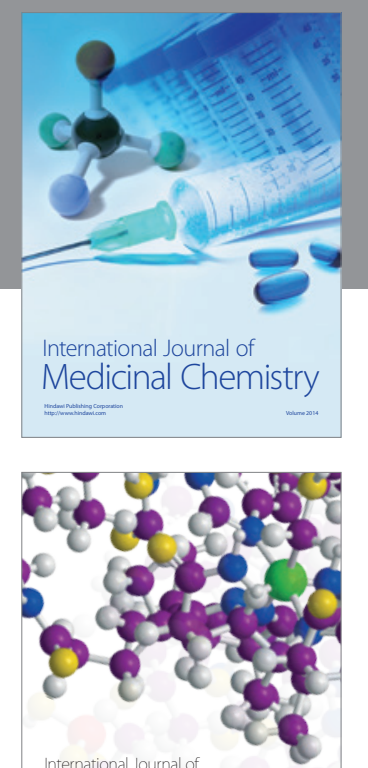

\section{Carbohydrate} Chemistry

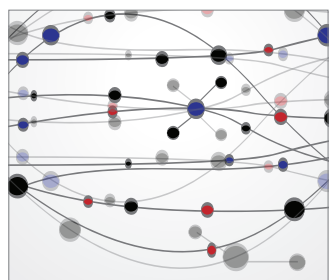

The Scientific World Journal
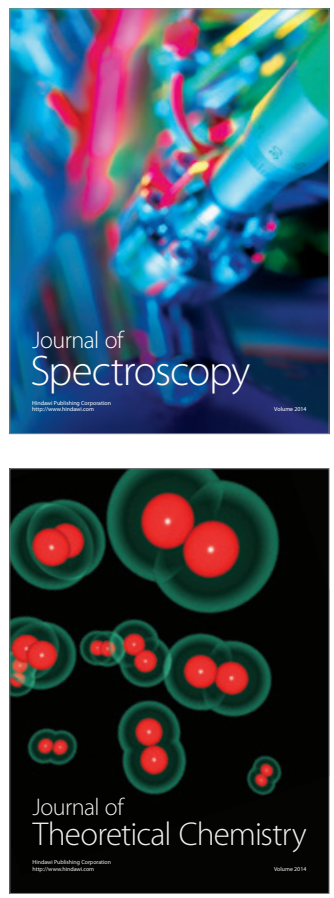
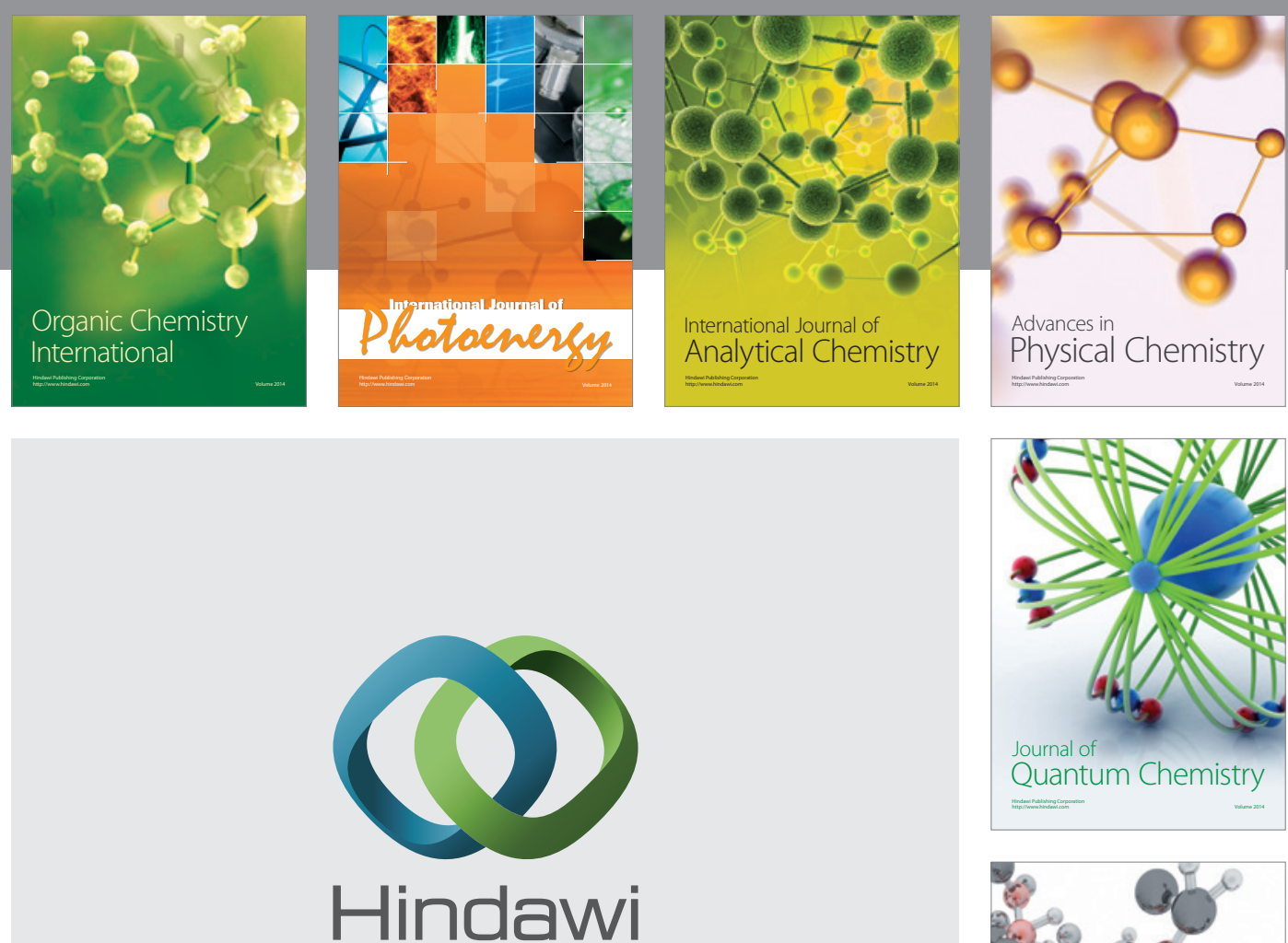

Submit your manuscripts at

http://www.hindawi.com

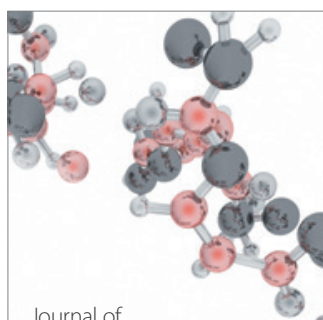

Analytical Methods

in Chemistry

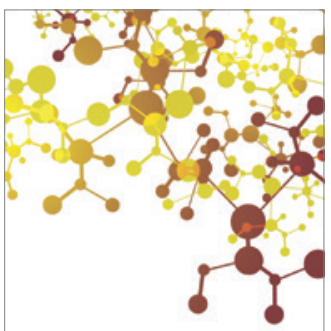

Journal of

Applied Chemistry

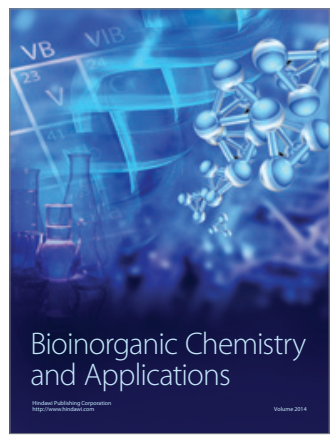

Inorganic Chemistry
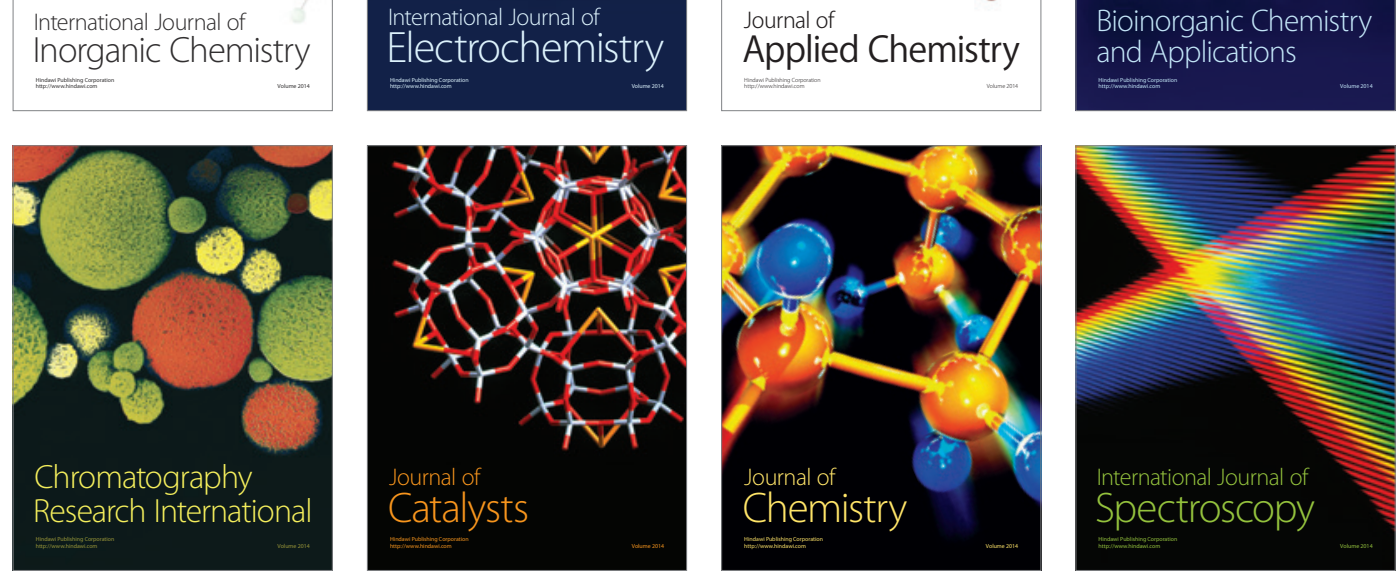